Pacific Journal of 


\title{
TWO THEOREMS ON GROUPS OF CHARACTERISTIC 2-TYPE
}

\section{GEOFFrey MASON}

\begin{abstract}
D. Gorenstein has made the following conjecture: suppose that $G$ is a finite simple group which is simultaneously of characteristic 2-type and characteristic 3-type. Then $G$ is isomorphic to one of $P S p(4,3), G_{2}(3)$ or $U_{4}(3)$. In this paper, we prove two results which, taken together, yield a proof of this conjecture under the additional assumption that $G$ has 2-local 3-rank at least 2.
\end{abstract}

1. Introduction. In this paper we study finite simple groups, all of whose 2-local and 3-local subgroups are 2-constrained and 3 -constrained respectively. The results we obtain are extensions of Thompson's theorem ES, and their relation to simple groups of characteristic 2-type is entirely analogous to the relation of theorem ES to simple $N$-groups.

The two Main Theorems are actually slight extensions of a conjecture of Gorenstein [10], and we refer the reader to [10] for a more detailed discussion of these ideas.

It will be convenient, before stating our main results, to develop some notation, most of which is standard.

Let $X$ be a group, $Y$ a subgroup of $X$, and $\pi$ a set of primes. Then $\boldsymbol{V}_{X}(Y ; \pi)$ denotes the set of $Y$-invariant $\pi$-subgroups of $X$. In particular, if the only $Y$-invariant $\pi$-subgroup of $X$ is 1 , we write $n_{X}(Y ; \pi)=\{1\}$.

For a finite group $X, \pi(X)$ is the set of prime divisors of $|X|$. As in [26], the subdivision of $\pi(X)$ into $\pi_{1}, \pi_{2}, \pi_{3}$ and $\pi_{4}$ will be important. We recall that $p \in \pi_{3} \cup \pi_{4}$ if a $S_{p}$-subgroup $P$ of $G$ has a normal abelian subgroup of rank at least 3 , which we write as $\operatorname{SCN}_{3}(P) \neq \varnothing$. Moreover,

$$
\begin{aligned}
& p \in \pi_{3} \text { if } \operatorname{SCN}_{3}(P) \neq \varnothing \text { and } \boldsymbol{U}_{X}\left(P ; p^{\prime}\right) \neq\{1\} \\
& p \in \pi_{4} \text { if } \operatorname{SCN}_{4}(P) \neq \varnothing \text { and } \boldsymbol{U}_{X}\left(P ; p^{\prime}\right)=\{1\} .
\end{aligned}
$$

If $p$ is a prime, $X$ a group, and $P$ a $S_{p}$-subgroup of $O_{p^{\prime}, p}(X)$, we say that $X$ is $p$-constrained if $C_{X}(P) \leqq O_{p^{\prime}, p}(X)$.

For $p$ a prime and $X$ a group, a $p$-local subgroup of $X$ is the normalizer of some nonidentity $p$-subgroup of $X$.

We say that $X$ is of characteristic $p$-type if $p \in \pi_{4}$ and every $p$-local subgroup of $X$ is $p$-constrained.

With these definitions we can now state Gorenstein's conjecture:

Suppose that $G$ is a finite simple group, $p$ an odd prime, and 
suppose further that $G$ is simultaneously of characteristic 2-type and characteristic $p$-type. Then $G$ is isomorphic to one of $G_{2}(3), P S p(4,3)$ or $U_{4}(3)$.

A little more notation is required to state our own results: if $p \in \pi_{3} \cup \pi_{4}, \mathfrak{N}(p)$ denotes the set of elementary abelian subgroups of type $(p, p)$ which are contained in elementary abelian subgroups of type $(p, p, p)$.

Suppose that $P$ is a $S_{p}$-subgroup of $X$ and that $\operatorname{SCN}_{3}(P) \neq \varnothing$. We write

$$
p \in \pi_{4}^{*} \quad \text { if } \quad{ }_{X}(P ; 2)=\{1\} .
$$

Hence we require that $P$ should not normalize any nontrivial 2-group, though it may well normalize some proper $\{2, p\}^{\prime}$-group.

We can finally state the main results of the present paper.

THEOREM 1. Suppose that $G$ is a finite simple group, $p$ an odd prime, and that the following conditions hold:

(a) $G$ is of characteristic 2-type.

(b) $p \in \pi_{4}^{*}$ and all p-local subgroups of $G$ are p-constrained.

(c) Some 2-local subgroup of $G$ contains an element of $\mathfrak{X}(p)$.

Then $p=3$ and $G$ is isomorphic to $G_{2}(3), P S p(4,3)$ or $U_{4}(3)$; or $p=5$ and $G$ satisfies the conditions of part (c) of Theorem D of [19].

THEOREM 2. Suppose that $G$ is a finite simple group and that the following conditions hold:

(a) $G$ is of characteristic 2-type.

(b) $3 \in \pi_{4}^{*}$ and all 3-local subgroups of $G$ are 3-constrained.

(c) Some 2-local subgroup of $G$ contains an elementary abelian subgroup of type $(3,3)$.

Then $G$ is isomorphic to $G_{2}(3), \operatorname{PSp}(4,3)$ or $U_{4}(3)$.

A word about the hypotheses of Theorems 1 and 2. Evidently hypothesis (b) is a little weaker than requiring $G$ to be of characteristic $p$-type (respectively, of characteristic 3-type). The point here is that the only relevant elements of $\eta_{G}\left(P ; p^{\prime}\right)$, for $P$ a $S_{p}$-subgroup of $G$, are the 2-groups. It is condition (c) which provides an initial hold on the subgroup structure of $G$. The strength of this assumption has previously been demonstrated in [19], and we will make use of the results obtained there in the present paper. Of course, elimination of (c) in Theorem 1 would provide a complete proof of Gorenstein's conjecture.

Finally we remark that although Theorems 1 and 2 are of a 
very similar nature, the proofs of the two theorems are completely different. Indeed if $G$ is a finite simple group of characteristic 2and $p$-type, the truth of Gorenstein's conjecture would imply that $p=3$. Under the assumptions of Theorem 1, the results of [19] already show that $p=3$, but in Theorem 2 we must assume at the outset that $p=3$. Moreover in Theorem 2 we have to account for the possibility that $G$ has a 2-local subgroup containing an elementary abelian subgroup $V \cong(3,3)$ with $V \notin \mathfrak{A}(3)$, and this causes difficulties. In view of Thompson's work in [4] and [26] this is not unexpected.

Finally, we emphasize that all groups considered in this paper are finite. Our notation is standard and usually follows that of [26]. We use the notation $Z_{n}, D_{n}, Q_{2} n, S D_{2} n$ to denote the cyclic group of order $n$, dihedral group of order $n$, generalized quaternion group of order $2^{n}$, and semidihedral group of order $2^{n}$ respectively. $A \backslash B$ is the regular wreathed product of $A$ with $B, A^{*} B$ the central product of $A$ and $B$, and $A \subseteq B$ means $A$ is isomorphic to a subgroup of $B$. Our notation for simple groups also follows [26], in particular $A_{n}, \sum_{n}$ are the alternating and symmetric groups of degree $n$ respectively. The solvability of groups of odd order [4] is assumed throughout.

2. Some preparatory lemmas. In this section we collect together some results which we shall need in the sequal. Most of the results are already in the literature.

Lemma 2.1 (Generalized $P \times$ Q-lemma): Suppose that $P$ is a p-group, $Q$ a q-group with $p$ and $q$ distinct primes and $p$ odd. Suppose further that $Q \triangleleft P Q$ and that $P Q$ normalizes the p-group $M$. Then $[Q, M]=1$ if, and only if, $\left[Q, \Omega_{1}\left(C_{M}(P)\right)\right]=1$.

Proof. This is contained in a result of Bender [2], and is a slight extension of a result of Thompson [9, Lemma 5.3.4] which we shall also need.

LEMMA 2.2. $P$ is a p-group which admits a fixed-point-free automorphism of order 3 . Then $P$ has class at most 2.

Proof. This is an old result of Burnside. A proof can be found in [12, Theorem 8.1].

Lemma 2.3. $G$ is a solvable group such that $O(G)=1$ and $\left|G: O_{2}(G)\right|=2.3^{a}, a \geqq 1$. Suppose that a $S_{3}$-subgroup $R$ of $G$ has $a$ subgroup $R_{0}$ of order 3 such that $R_{0}$ is weakly closed in $R$ and 
$C\left(R_{0}\right) \cap O_{2}(G)=1$. Then one of the following holds:
(a) $\mathrm{G} \cong \sum_{4}$
(b) $\mathrm{O}_{2}(G)$ is characteristic in a $S_{2}$-subgroup of $G$.

Proof. Let $Q=Q_{2}(G)$ with $T$ a $S_{2}$-subgroup of $G$, so that $|T: Q|=2$. If $|Q|=4$ then (a) obviously holds, so we may assume that $|Q| \geqq 8$. Now as $C_{Q}\left(R_{0}\right)=1$ then $Q$ has class at most 2 by Lemma 2.3. We will show that $Q$ is the only subgroup of $T$ of its isomorphism class, in which case (b) is immediate.

Now by the Frattini argument we have $G=Q N_{G}(R)$, so because of the existence of $R_{0}$ we get $\left|N_{G}(R)\right|=2|R|$, so $N_{G}(R)=R\langle t\rangle$ with $T=Q\langle t\rangle$ for some involution $t$. Since $R_{0}$ is weakly closed in $R$ then $R_{0} \triangleleft R\langle t\rangle$ so $t$ either inverts or centralizes $R_{0}$. If $t$ inverts $R_{0}$ then the desired result is proved by Higman in [12, Theorem 8.1], so we may assume that $\left[R_{0}, t\right]=1$.

Suppose to begin with that $Q$ is abelian. If there is a second subgroup of $T$ which is isomorphic to $Q$ then $t$ must centralize a subgroup of index 2 in $Q$. As $R_{0}$ normalizes $C_{Q}(t)$ we get $Q \cap C\left(R_{0}\right) \neq 1$, a contradiction. Now suppose that $Q$ has class 2. Higman shows in [12, Theorem 8.1] that every subgroup $Q_{0}$ of $Q$ with $\left|Q: Q_{0}\right|=2$ is such that $Q^{\prime}=Q_{0}^{\prime}$. If there is a subgroup $Q_{1}<T$ with $Q_{1} \cong Q$ and $Q_{1} \neq Q$ then $Q_{0}=Q_{1} \cap Q$ has index 2 in $Q$, so we get $Q_{0}^{\prime}=Q^{\prime}$. But $Q_{0}^{\prime} \leqq Q_{1}^{\prime} \cong Q^{\prime}$, so $Q_{1}^{\prime}=Q^{\prime}$. Finally, set $\bar{G}=G / Q^{\prime}$. As $Q^{\prime} \leqq \phi(Q)$ then $\bar{G} / \bar{Q}$ acts faithfully on $\bar{Q}$ and $\bar{Q}$ is abelian. Since $Q_{1}=Q_{0}\langle t q\rangle$ for some $q \in Q$ and $\bar{Q}_{1}$ is abelian then $\bar{t}$ centralizes $\bar{Q}_{0}$. But $\left|\bar{Q}: \bar{Q}_{0}\right|=2$, so $\bar{t}$ centralizes a subgroup of $\bar{Q}$ of index 2 and we obtain a contradiction as before. This completes the proof of Lemma 2.3.

The next result, though apparently of an elementary nature, requires deep results of Gorenstein and Gilman [5] and Walter [27] for its proof.

LEMMA 2.4. G is a 3'-group which admits an automorphism a of order 3 such that $C_{G}(a)$ has odd order. Then $G$ is solvable of 2-length 1.

Proof. Since $(|G|,|\langle a\rangle|)=1$ then $G$ has an $\langle a\rangle$-invariant $S_{2}$-subgroup $T$. By Lemma $2.2 T$ has class at most 2 .

First we show that $G$ is solvable, so suppose that this is not the case. Proceeding by induction, we may assume that $G$ is characteristically simple, hence is the direct product of isomorphic groups $G_{1}, \cdots, G_{r}$. As $\langle a\rangle$ permutes the $G_{i}$ among themselves we get $r=1$ or 3 and if $r=3$ and then $\langle a\rangle$ is transitive on $\left\{G_{1}, G_{2}, G_{3}\right\}$. But in this case $\langle a\rangle$ must centralize the involution $t t^{a} t^{a^{2}}$ whenever $t$ is an involution of $G_{1}$, against the assumption that $C_{G}(a)$ has odd order. 
So in fact $G$ is simple. As $T$ has class at most 2 we may identify $G$ by the results of Gorenstein-Gilman and Walter mentioned above. As $G$ is $3^{\prime}$-group the only possibility $G \cong S z(q)$ for some $q \geqq 8$. But every outer automorphism of $S z(q)$ has a fixed-point subgroup of even order (see [25]), which contradiction completes the proof that $G$ is solvable.

In proving that $G$ has 2-length 1 we may assume that $O(G)=1$ and try to prove that $T \triangleleft G$. As $G$ is solvable it has an $\langle a\rangle$-invariant Hall 2'-subroup $H$, and $H\langle a\rangle$ acts faithfully $Q=O_{2}(Q)$. Next we show $[a, H]=1$, so suppose this is not the case and choose $V$ to be an $\langle a\rangle$-invariant subgroup of $H$ minimal subject to $[V, a] \neq 1$. As $V\langle a\rangle$ is faithful on $Q$, it is well known that $C_{Q}(a) \neq 1$, a contradiction. So $V$ does not exist and hence $[H, a]=1$. It follows that $Q\langle a\rangle \triangleleft$ $G\langle a\rangle$, and hence $T=Q N_{T}(\langle a\rangle)$. But $N_{T}(\langle a\rangle)=C_{T}(\alpha)=1$, so $Q=T$ as required.

LeMma 2.5. Gis a simple group with a cyclic $S_{p}$-subgroup $P, p$ an odd prime. Suppose that $C(P)$ has odd order and $|N(P): C(P)|=2$. Then $G$ has only one class of icvolutions.

Proof: This is a result of Brauer's [3].

Lemma 2.6. $G$ is a group, $p$ an odd prime, $G=O^{P}(G)$, and $P$ a $S_{p}$-subgroup of $G$. Suppose that $P$ is non-cyclic and abelian, and that $|N(P): C(P)|=2$. Then $G$ is p-solvable.

Proof. This is a recent result of Smith and Tyrer [24].

Lemma 2.7. $G$ is a simple group of characteristic 2-type. Then $O(N)=1$ for each 2-local subgroup $N$ of $G$.

Proof. This is a well-known result of Gorenstein [7] which we will frequently use without specific reference to it.

LEMMA 2.8. $G$ is a simple group of characteristic 2-type with a maximal 2-local subgroup $N$ such that $O_{2}(N)$ is of sympletic type. Then $G$ has a non-solvable 2-local subgroup.

Proof. This is contained in a result of Lundgren [20]. (Observe that $O(N)=1$ by Lemma 2.7.)

LEMmA 2.9. $G$ is a simple group such that $2 \in \pi_{3} \cup \pi_{4}$ and $C_{G}(x)$ is solvable of 2-length 1 for each involution $x$ of $G$. Then $G$ is isomorphic to one of the following groups: $L_{2}(q), S z(q)$ or $U_{3}(q)$ for $q=2^{n} \geqq 8$. 
Proof. This is a combination of results of Bender, Goldschmidt and Suzuki. The result is discussed in [8].

Lemma 2.10. $G$ is a simple group and $T$ a $S_{2}$-subgroup of $G$. Suppose that $T$ has an abelian subgroup of index at most 2. Then the following hold:

(a) $T$ is either abelian or isomorphic to $D_{2} n, S D_{2} n$ or $Z_{2} n \geq Z_{2}$.

(b) $G$ is isomorphic to one of the following groups: $L_{2}(q), q \geqq 4$, $L_{3}(q)$ or $U_{3}(q), q \geqq 3$, a group of Ree-type, $A_{7}, M_{11}$ or $J_{1}$.

Proof. This is the combination of the work of a number of authors. For a fuller discussion of the result we refer the reader to $[21]$.

3. The Proof of Theorem 1. In this section we will present a proof of Theorem 1. So for the balance of this section $G$ will denote a simple group satisfying the hypotheses of Theorem 1 , and $p \neq 5$.

We have already made an initial investigation of the consequences of the hypotheses of Theorem 1 in some joint work with Klinger [19]. We obtained there the following result which represents the first major reduction in the proof of Theorem 1.

Proposition 3.1. Under the assumptions of Theorem 1, the following conditions hond.

(a) $p=3$

(b) No 2-local subgroup of $G$ contains an elementary abelian subgroup of type $(3,3,3)$.

(c) We can choose $B \in \mathfrak{N}(3)$ and a maximal element $F$ of $\mathrm{U}(B ; 2)$ such that $F$ is extra-special of width $w \leqq 4$. Moreover $F$ is the central product of $w$ B-invariant quaternion subgroups. We have $Z=Z(F)=C_{F}(B)$, and $C_{F}\left(B_{0}\right)$ has rank 1 for $1<B_{0}<B$.

We shall retain the notation of Proposition 3.1 throughout. Moreover we set $\widehat{B}=\left\{B_{0} \mid 1<B_{0}<B\right.$ and $\left.C_{F}\left(B_{0}\right) \neq Z\right\}$. Thus $\mathrm{C}_{F}\left(B_{0}\right) \cong Q_{8}$ for $B_{0} \in \hat{B},|\hat{B}|=w$, and if $\hat{B}=\left\{B_{1}, \cdots, B_{w}\right\}$ with $C_{F}\left(B_{\imath}\right)=Q_{i}, 1 \leqq i \leqq w$, then $F=Q_{1}^{*} \ldots * Q_{w}$. We also set $Z=\langle z\rangle$.

We also obtained in [19] the following useful result.

Lemma 3.2. Suppose that $D \in \mathfrak{V}(3), H \in \mathcal{U}(D ; 2)$, and $\boldsymbol{C}_{H}(D) \neq 1$. Then $H$ is of symplectic-type.

Lemma 3.3. Let $x$ be an involution of $G$. Then exactly one of the following holds. 

(a) $C_{G}(x)$ has cyclic $S_{3}$-subgroups.
(b) $x \sim z$ in $G$.

Proof. Suppose that (a) is false, in which case $C(x)$ contains a noncyclic elementary 3-subgroup $D$. By Proposition 3.1(b) we have $|D|=9$. Suppose to begin with that $D \in \mathfrak{A}(3)$. Then if $\langle x\rangle \leqq$ $F_{0} \in U^{*}(D ; 2)$ we get that $F_{0}$ is of symplectic-type by Lemma 3.2 , in particular $\langle x\rangle=\Omega_{1}\left(Z\left(F_{0}\right)\right)$. But if $T$ is a $S_{2}$-subgroup of $G$ containing $F_{0}$ we must have, since $O_{2}\left(N\left(F_{0}\right)\right)=F_{0}$, that $Z(T)<F_{0}$. Hence $\langle x\rangle=\Omega_{1}(Z(T))$ and so $x \sim z$.

Finally, suppose that $D \notin \mathfrak{U}(3)$. We will show that this case cannot occur: namely, if $D \notin \mathfrak{A}(3)$ then $D$ contains every element of order 3 in $C(D)$, in particular if $R$ is a $S_{3}$-subgroup of $G$ containing $D$ then $Z_{0}=\Omega_{1}(Z(R))<D$. Set $L=C_{G}\left(Z_{0}\right)>R$. As $S C N_{3}(R) \neq \varnothing$ then $O_{3}(L)$ has odd order, so $x$ acts faithfully $O_{3^{\prime}, 3}(L) / O_{3 \prime}(L)$. Set $P=O_{3^{\prime}, 3}(L) / O_{3}(L) .\langle x\rangle \times D$ acts on $P$ and we have $\left[x, \Omega_{1}\left(C_{P}(D)\right)\right] \leqq$ $[x, D]=1$, hence $\left[x, C_{P}(D)\right]=1$, hence $[x, P]=1$ by the $P \times Q$-lemma. This contradiction proves the lemma.

We now set $C=C_{G}(Z)$. $T$ will denote some fixed $S_{2}$-subgroup of $C$, and $R$ is a $S_{3}$-subgroup of $C$ which contains $B$. We collect the facts we shall need about $C$ in the following lemma:

Lemma 3.4. The following conditions hold:

(a) $C=N(F)$, and $C$ is a maximal 2-local subgroup of $G$.

(b) $T$ is a $S_{2}$-subgroup of $G$ and $Z=Z(T)$.

(c) Either $R=B$ is elementary of order 9 or one of the following holds:

(i) $w=3$ and $R$ is non-abelian of order 27 .

(ii) $w=4$ and $R$ is non-abelian metacyclic of order 27 .

(d) $\boldsymbol{h}_{C}^{*}\left(B ; 3^{\prime}\right)=\{F\}$.

Proof. We have $|Z|=2$, so clearly $N(F) \leqq C$. On the other hand if $F_{0}=O_{2}(C)$ we must have $F_{0} \leqq F$ since otherwise we get $F<F_{0} F \in h(B ; 2)$, against the maximality of $F$. Now as $C$ is 2-constrained we must have $Z<F_{0}$, hence $C_{F_{0}}(B) \neq 1$ and $F_{0}$ is of symplectic-type by Lemma 3.2. We deduce that $F_{0}$ is a product of the subgroups $Q_{1}, \cdots, Q_{w}$. If $F_{0} \neq F$ then some $Q_{i}$, say $Q_{1}$, satisfies $Q_{1} \cap F_{0}=Z$. But then $\left[Q_{1}, F_{0}\right]=1$, against the 2-constraint of $C$, and so we must have $F_{0}=F$ and $N(F)=C$. The same proof now yields that $C$ is also a maximal 2-local subgroup of $G$, so (a) is proved. (b) is a straight forward consequence of (a).

As for (c), suppose that $B<R$. In this case, we must have $w=3$ or 4 , so assume to begin with that $w=3$. Hence, we get $C / F \subseteq$ Out. $(F) \cong O_{6}^{-}(2)$, so $R G Z_{3} \succ Z_{3}$. Since $R$ has rank 2 by 
Proposition 3.1(b) we get that $R$ is non-abelian of order 27. Now suppose that $w=4$ and assume without loss that $B_{1} \leqq B \cap Z(R)$. Hence $R$ normalizes $Q_{1}=C_{F}\left(B_{1}\right)$ and $C_{R}\left(Q_{1}\right)=R_{1}$ is a cyclic subgroup of $R$ index 3, in particular $R$ is metacyclic. Moreover $R_{1}$ is faithful on $Q_{2}^{*} Q_{3}^{*} Q_{4}$ so $R_{1} \subseteq Z_{3} \succ Z_{3}$ and $\left|R_{1}\right|=9$. This completes the proof of (c).

Now we certainly have $\{F\}=\boldsymbol{U}_{C}^{*}(B ; 2)$ by part (a), so to prove (d) it suffices to show that $\boldsymbol{U}_{C}(B ; q)=\{1\}$ for each prime $q \in\{2,3\}^{\prime}$. We get, since $q \geqq 5$, that $\left[C_{Q}(B), Q_{i}\right]=1$ for $1 \leqq i \leqq w$, hence $\left[C_{Q}(B), F\right]=1$, hence $C_{Q}(B)=1$ by 2-constraint. Now if $w=3$ we get $|Q|=5$ and hence $[Q, B]=1$, a contradiction. Thus $w=4$. Now choose $b \in B^{\sharp}$ with $C_{Q}(b) \neq 1$. We have $C_{F}(b) \cong Q_{8}$ so $\left[C_{Q}(b)\right.$, $\left.C_{F}(b)\right]=1$, hence $\left|C_{Q}(b)\right|=5$ and we get $\left[C_{Q}(b), B\right]=1$ which is again impossible. This completes all parts of the lemma.

As a corollary of Lemmas 3.4(a) and 2.8 we obtain immeditely

Lemma 3.5. G has a non-solvable 2-local subgroup.

Next we prove

LEMma 3.6. One of the following occurs.

(a) $C$ is solvable.

(b) $w=4$ and $C / F$ is isomorphic to a subgroup of $\operatorname{Aut}\left(A_{6}\right)$ containg Inn. $\left(A_{6}\right)$.

Proof. Suppose that $C$ is nonsolvable. Then we obviously have $w \geqq 3$. Suppose that $w=3$, and let $E / F$ be a minimal normal subgroup of $C / F$. By Lemma 3.4(d) $E$ has order divisible by 3, so as all 3 -local subgroups of $O_{6}^{-}(2) \cong$ Out. $(F)$ are solvable whilst $C$ is nonsolvable we must have that $E / F$ is simple. Now $O_{6}^{-}(2)$ has 3 -rank 3 , whilst $C$ has 3 -rank 2 by Proposition 3.1(b). We deduce that $E / F \not$ $O_{6}^{-}(2)$, so the only possibilities are $E / F \cong A_{5}$ or $A_{\hat{6}}$. But if $E / F \cong A_{5}$ some element of $B^{\sharp}$ centralizes $E / F$, an impossibility. Suppose that $E / F \cong A_{6}$. In this case $B$ is a $S_{3}$-subgroup of $E$ and there is a cyclic subgroup of order 4 in $E / F$ normalizing and acting irreducibly on $B$. On the other hand there is exactly one subgroup of $B$ of order 3 not in $\hat{B}$, so $N_{E}(B)$ cannot act irreducibly on $B$. This contradiction establishes that fact that $w=4$ if $C$ is nonsolvable.

Again let $E / F$ be a minimal normal subgroup of $C / F$, where we are now assuming that $w=4$. As before $E / F$ is either simple or a 3-group. If the latter case occurs then we can choose $b \in(E \cap B)^{*}$. Setting $\bar{C}=C / F$ we deduce that $C_{\bar{C}}(\bar{b})$ is nonsolvable and that $C_{\bar{C}}(\bar{b})$ has a normal subgroup $\bar{A} \cong A_{5}$. But then $\bar{B}$ normalizes a $S_{3}$-subgroup of $\bar{A}$, against $F \in h^{*}(B ; 2)$, so we have shown that $E / F$ is simple. 
Next, let $S$ be a $S_{7}$-subgroup of $E$ and suppose that $S \neq 1$. Thus $|S|=7$, and a simple computation shows that $C_{F}(S) \cong D_{8}$. Now as no 3-element of $\bar{C}^{\ddagger}$ contralizes a four-group of $F$ and as $O_{8}^{+}(2)$ has no elements of order 35 we deduce that $N_{C}(S)$ is a $\{2,7\}$-group. A Frattini argument now yields $R<E$, so $R=B$ by Lemma 3.4(c) and a theorem of Huppert [14]. Sylow's theorem now tells us that $|E: F|=2^{a} 3^{27}$ or $2^{a} 3^{2} 5^{27}$ for some $a$. However, $O_{8}^{+}(2)$ has no such simple subgroup (with elementary $S_{3}$-subgroups), so we have shown that $E$ is a $7^{\prime}$-group. Being simple $E / F$ is a $\{2,3,5\}$-group, and surveying the possibilities we find that $E / F \cong A_{5}$ or $A_{6}$. However if $E / F \cong A_{5}$ then some element of $B^{\ddagger}$ contralizes $E / F$, and we have already shown that this cannot occur. Hence $E / F \cong A_{6}$, and the conclusions of Lemma 3.6 now follows easily.

Lemma 3.7. Suppose that $w=3$ and that $B_{0}$ is the subgroup of $B$ of order 3 satisfying $B_{0} \notin \hat{B}$. Then the following hold:

(a) $C_{C}\left(B_{0}\right)$ has cyclic $S_{2}$-subgroups.

(b) $C$ contains a $S_{2}$-subgroup of $C_{G}\left(B_{0}\right)$.

Proof. Let $I$ be a $S_{2}$-subgroup of $C_{C}\left(B_{0}\right)$. Thus $Z \leqq I$. Since $B_{0} \notin \hat{B}$ we have $I \cap F=Z$. Now if (a) is false then there is an involution $x \in I-Z ; x$ must invert a subgroup of $\hat{B}$ so we may suppose that $x$ inverts $B_{1}$. Thus $\langle x\rangle \times B_{0}$ normalizes $Q_{1}=C_{F}\left(B_{1}\right)$, so $\left[x, Q_{1}\right]=1$ since $B_{0}$ is faithful on $Q_{1}$.

Next, suppose that either $x$ or $x z$ (say $x$ for definiteness) is conjugate to $z$, and set $L=C_{G}(x)$. We have $\langle x\rangle=\phi\left(O_{2}(L)\right)$ and $B_{0} Q_{1}<L$. Since $L \cong C$ and $Q_{1}=\left[Q_{1}, B_{0}\right]$ we get $Q_{1}<O_{2}(L)$, hence $Z=\phi\left(Q_{1}\right)=\langle x\rangle$. This is false, so we deduce that neither $x$ or $x z$ is conjugate to $z$.

Finally consider $N=N_{G}\left(B_{1}\right)>B_{1}\left(Q_{1} \times\langle x\rangle\right)$. If $P$ is an $\langle x, z\rangle-$ invariant $S_{3}$-subgroup of $O_{3^{\prime}, 3}(N)$ then $P=\left\langle C_{P}(y) \mid y \in\langle x, z\rangle^{\sharp}\right\rangle$. But by Lemma 3.3 and the provious paragraph we get that both $C_{P}(x)$ and $C_{P}(x z)$ are cyclic, hence are both centralized by $z$. But then $z$ centralizes $P$, contradiction. This proves part (a) of the lemma, and (b) is a straightforward consequence of it.

Lemma 3.8. Suppose $w \geqq 3$. Then $C$ controls fusion of its subgroups of order 3.

Proof. As a consequence of Lemma 3.7(b) we get (with the notation of that lemma) that $B_{0}$ is conjugate to no element of $\hat{B}$ in case $w=3$. Now suppose that $B_{5}, B_{6}$ are two subgroups of $R$ of order 3 with $B_{5} \sim B_{6}$ in $G$. It follows that $C_{F}\left(B_{i}\right)$ is a quaternion group, $i=5,6$. 
Let $Q_{2}=C_{F}\left(B_{i}\right)$ with $I_{i}$ a $S_{2}$-subgroup of $C_{C}\left(B_{i}\right), i=5,6$. Thus $Q_{i} \unlhd I_{i}$. A simple computation shows that $I_{i}$ is either quaternion or semidihedral (of order 16), so in any case we have $Z=Z\left(I_{i}\right)$ and $I$, is a $S_{2}$-subgroup of $C_{G}\left(B_{i}\right), i=5,6$. As $B_{5} \sim B_{6}$ we can choose $g \in G$ satisfying $B_{5}^{g}=B_{6}, I_{5}^{g}=I_{6}$ by Sylow's theorem. Hence $Z^{g}=Z$, that is $g \in C$, as required.

Lemma 3.9. Suppose $w \geqq 3$ and $x$ is a involution with $x \in C-F$, Then $C_{C}(x)$ is a 2-group.

Proof. We only need show, after Lemmas 3.4 and 3.6, that $x$ centralizes no nontrivial 3-subgroup of $C$, so suppose that this is false. By Lemma 3.7 we can assume that $x$ centralizes $B_{1}$. We calculate that $\left\langle x, Q_{1}\right\rangle$ is semidihedral of order 16 .

Now set $N=N_{G}\left(B_{1}\right)$, and let $P$ be a $\left\langle x, Q_{1}\right\rangle$-invariant $S_{3}$-subgroup of $O_{3^{\prime}, 3}(N)$. Thus $P=\left\langle C_{P}(y) \mid y \in\langle x, z\rangle^{\sharp}\right\rangle$. If $x \neq z$ in $G$ then $C_{P}(x)=C_{P}(x z)$ is cyclic by Lemma 3.3, hence $B_{1}=\Omega_{1}\left(C_{P}(x)\right)=\Omega_{1}\left(C_{P}(x z)\right)$, hence $z$ centralizes $P$, contradiction. It follows that $x \sim x z \sim z$ in $G$. Setting $L=C_{G}(x)$ and $x=z^{g}$, we get $B_{1}, B_{1}^{g}<L$, so $B_{1}=B_{1}^{g^{1}}$ for some $1 \in L$ by Lemma 3.8. We have thus shown that $x \sim x z \sim z$ in $N$.

Finally, since $C_{P}(Z)$ admits $Q_{1}$ we must have $B_{1}=\Omega_{1}\left(C_{P}(Z)\right)$, so $B_{1}=\Omega_{1}\left(C_{P}(y)\right)$ for any $y \in\langle x, z\rangle^{\sharp}$. This is absurd, and the lemma is proved.

LEMMA 3.10. The following conditions hold.

(a) If $B_{i} \in \hat{B}$ then $C_{F}\left(B_{i}\right)$ is a $S_{2}$-subgroup of $C_{C}\left(B_{i}\right)$.

(b) If $C$ is solvable and $w \geqq 3$ then $C / F$ has cyclic $S_{2}$-subgroups.

(c) If $C$ is nonsolvable then $C / F$ has 2-rank 2.

Proof. To prove (a), let $B_{i} \in \hat{B}$ with $Q_{i}=C_{F}\left(B_{\imath}\right)$ and suppose that $Q_{i}$ is not a $S_{2}$-subgroup of $C_{C}\left(B_{\imath}\right)$. Let $I_{i}$ be a $S_{2}$-subgroup of $C_{c}\left(B_{i}\right)$ which contains $Q_{i}$. By Lemma $3.9 I_{i}$ is generalized quaternion of order at least 16 , so $C_{C}\left(B_{i}\right)$ has a normal 2-complement. But then we get $\left[B, Q_{i}\right] \leqq Q_{i} \cap O\left(C_{C}\left(B_{i}\right)\right)=1$, a contradiction which proves (a).

(b) is a simple consequence of (a) together with Lemma 3.7(a).

Finally, suppose that $C$ is nonsolvable and that $C / F$ has 2-rank at least 3. By Lemma 3.6 we must have that $C / F$ contains a subgroup isomorphic to $\sum_{6}$, and that $w=4$. But $\sum_{6}$ has elements of order 6 and every subgroup of $B$ of order 3 lies $\hat{B}$ as $w=4$. This contradicts (a), and the lemma is proved.

LEMma 3.11. Suppose that $w \geqq 3$. Then $Z$ is weakly closed in $F$. 
Proof. Suppose false. Then there is $g \in G-C$ such that $Z^{g}=$ $Y<F$. Set $C^{g}=L=C_{G}(Y)$ with $D=O_{2}(L)$. Now $C_{F}(Y)=Y \times F_{0}$ where $F_{0}$ is extra-special of width $w-1$ and $F_{0}<L$. It is a simple consequence of Lemma 3.10 that $F_{0} \cap D \neq 1$, so $Z<D$, so $F_{0} D / D$ is elementary abelian. By Lemma 3.10 again, we deduce that $\left|F_{0}: F_{0} \cap D\right| \leqq 4$, and $\left|F_{0}: F_{0} \cap D\right| \leqq 2$ if $w=3$ (for $C$ is solvable in this case). Thus $F_{0} \cap D$ is not elementary abelian, so we obtain the contradiction $Z=\dot{\phi}\left(F_{0} \cap D\right)=\dot{\phi}(D)=Y$. This proves the lemma.

It is now easy to prove

\section{Proposition 3.2. The case $w=3$ cannot occur.}

Proof. Let $N$ be a nonsolvable 2-local subgroup of $G$, the existence of which is guaranteed by Lemma 3.5, and set $D=O_{2}(N)$ with $N_{2}$ a $S_{2}$-subgroup of $N$. As $w=3$ then $C$ is solvable by Lemma 3.6, so $N \neq C$. Lemma 3.4(b) allows us to assume that $N_{2}<C$, in which case $Z<Z(D)$.

Let $V=\Omega_{1}(Z(D))$, so that $Z<V \triangleleft N$. As $w=3$ Lemma 3.10(b) shows that a $S_{2}$-subgroup of $C$ has rank at most 4 , in particular $V \mid \leqq 16$. So if $\mathfrak{X}$ is the $N$-orbit of $V^{\ddagger}$ which contains $z$ then $|\mathfrak{X}| \leqq 9$, as follows from Lemma 3.11.

Now we have $|N|=|\mathfrak{X}|\left|C_{N}(Z)\right|$. Since $C_{N}(Z)$ is a $\{2,3\}$-group, $N$ is nonsolvable, and $|\mathfrak{X}| \leqq 9$, we deduce that $|\mathfrak{X}|=5$ or 7 . In either case there is a subgroup $J<N$ of order 3 centralizing $Z$, so $J<C$. Since $V=C_{V}(J)[V, J]$, Lemmas 3.9 and 3.10 yield $V<F$, so $\mathfrak{X} \subseteq F$ against Lemma 3.11. This completes the proof of Proposition 3.2.

Proposition 3.3. The case $w=4$ cannot occur.

Proof. Our proof in this case is a little different to that of Proposition 3.2, since if $C$ is nonsolvable, then Lemma 3.5 is of no help. We break the proof into a number of steps. We start with

(1) Suppose that $x$ is an involution of $C-F$, and that $x \in O^{2}(C)$ if $C$ is nonsolvable. Then $C_{F}(x)$ is elementary of order 16 .

Observe that since $w=4$ then every subgroup of order 3 in $B$ lies in $\hat{B}$. Now as $x \notin O_{2}(C)$ then $x$ inverts a subgroup of $C$ of prime order by the Baer-Suzuki theorem [1]. If $C$ is solvable such a subgroup must have order 3 by Lemma 3.4(c). If $C$ is not solvable then in $\bar{C}=C / F, \bar{x}$ must invert a subgroup of order 3 by the structure of $A_{6}$. Let $B_{0}$ be a subgroup of $C$ such that $\bar{x}$ inverts $\bar{B}_{0}$. Thus $H=F B_{0}\langle x\rangle$ is a group. If $H$ is 2-closed we get that $\left|C_{H}\left(B_{0}\right)\right|_{2}=16$, so $C_{F}\left(B_{0}\right)$ is not a $S_{2}$-subgroup of $C_{C}\left(B_{0}\right)$, against Lemma 3.10(a). So $H$ is not 2-closed, hence $x \notin O_{2}(H)$, hence $x$ inverts a subgroup of $H$ of order 3. So in any case $x$ inverts a subgroup of $C$ of order 3, 
and we may assume that such a subgroup is $B_{1} \in \hat{B}$.

The same argument also shows that $x$ inverts a subgroup of order 3 in $C_{c}\left(B_{1}\right) / B_{1}$, so that $x$ inverts an elementary subgroup of $C$ of order 9 . We may therefore assume that $x$ inverts $B$. Hence $x$ normalizes $Q_{i}$ and $\left\langle Q_{i}, x\right\rangle \cong S D_{16}$ for $1 \leqq i \leqq 4$. A simple computation now proves (1).

(2) Let $y$ be an involution of $F-Z$ such that $C$ contains a $S_{2}$-subgroup of $C_{G}(y)$. Then $C_{G}(y)<C$.

Set $Y=\langle y\rangle, L=C_{G}(Y), D=O_{2}(L)$. Since $D<C$ we get $Z<V=$ $\Omega_{1}(Z(D))$. Proceeding under the assumption that $L \nless C$ we get $Z \nless L$, so if $\mathfrak{X}$ is the $L$-orbit of $V^{*}$ which contains $z$ we get that $|\mathfrak{X}|$ is odd and $|\mathfrak{X}| \geqq 3$. By Lemma 3.11 we get $\mathfrak{X} \cap F=\{z\}$.

We claim next that $D \cap F$ is elementary abelian. If $C$ is solvable or $C$ is nonsolvable and $(\mathfrak{X}-\{z\}) \cap O^{2}(C) \neq \varnothing$, this is an immediate consequence of step (1), so we may suppose that neither of these conditions hold. Choose $x \in \mathfrak{X}-\{z\}$, Thus by Lemma 3.6(b) and 3.10(c) we have $C=O^{2}(C)\langle x\rangle$. In this case we have that $x \notin \phi(K)$ whenever $K$ is a 2-subgroup of $C$ containg $x$, in particular $x \in \phi(D)$. As $x \sim z$ in $L$ then also $z \notin \dot{\phi}(D)$, so $z \notin \phi(D \cap F)$ so $D \cap F$ is elementary abelian, as required.

Now $C_{F}(Y)=Y \times F_{0}$ where $F_{0}$ is extra-special of width 3 . By the last paragraph $D \cap F_{0}$ is elementary, so $\left|F_{0}: D \cap F_{0}\right| \geqq 8$, so $L / D$ has 2-rank at least 3.

Next we show that $C_{L}(Z)$ is a 2-group. If false, the structure of $C$ yields only one possibility, namely that $C_{L}(Z)$ is a $\{2,5\}$-group and $C$ is nonsolvable. Let $K$ be a $S_{5}$-subgroup of $C_{L}(Z)$, so that $V=C_{V}(K) \times[V, K]$. Now it is a simple consequence of step (1) that $|V| \leqq 2^{6}$, so if $[V, K] \neq 1$ then $|[V, K]|=2^{4}, C_{V}(K)=\langle Y, Z\rangle$, so $V<F$. This is false, so $[V, K]=1$ and $K<C_{L}(V) \triangleleft L$. However $\left[F_{0}, K\right]$ is nonabelian and $\left[F_{0}, K\right] \leqq O_{2}\left(C_{L}(V)\right)=O_{2}(L)$. This is impossible as $D \cap F$ is elementary, so we have shown that $C_{L}(Z)$ is a 2-group.

Since $|L|=|\mathfrak{X}|\left|C_{L}(Z)\right|$ we deduce that $|\mathfrak{X}|=|L|_{2^{\prime}}<63$. As 3.5.7 $>63$ it follows that $\pi(L)$ contains at most 3 distinct primes. Next, suppose that $L$ is solvable. As $L / D$ has 2-rank at least 3 and $L$ has cyclic $S_{3}$-subgroups by Lemma 3.3 , we gain a simple contradiction using Lemma 5.34 of [26]. So $L$ is nonsolvable. Hence $L / D$ is a nonsolvable $\{2,3, p\}$-group with cyclic $S_{3}$-subgroups, 2-rank at least $3, O_{2}(L / D)=1,|L: D|_{2^{\prime}}<63$, and $L / D G G L(5,2)$. There are no such groups, and step (2) is proved.

(3) If $y$ is an involution of $F-Z$ then $C_{G}(y)<C$. If $C$ contains a $S_{2}$-subgroup of $C_{G}(y)$ we are done by step (2). In any case by Sylow's theorem $y$ is conjugate to an involution $x$ of $C$ such that $C$ contains a $S_{2}$-subgroup of $C_{G}(x)$. Suppose that $x^{\sigma}=y$ for 
some $g \in G$. Evidently $x \in F$, so we get $C(y)<C^{g}$ by step 2. Now if $C_{F}(y)=\langle y\rangle \times F_{0}$ then $F_{0}$ is extra-special of width 3. By the structure of $C^{g}$ we get $F_{0} \cap O_{2}\left(C^{g}\right) \neq 1$, so $Z<O_{2}\left(C^{g}\right)$, so $Z=Z\left(O_{2}\left(C^{g}\right)\right)$ by Lemma 3.11. Hence $C^{g}=C$, and step (3) follows.

(4) $Z$ is weakly closed in $C$ with respect to $G$. For suppose that $g \in G$ and $Z^{g}<C, Z^{g}=Y \neq Z$. Set $L=C_{G}(Y), D=O_{2}(L)$. A straightforward calculation shows that $F \cap D \neq 1$, so let $f$ be an involution with $f \in F \cap D$. By step 3 we get $C_{G}(f) \leqq L$, in particular $C_{F}(f)<L$. This leads to $Z<D$, so $Z=Z\left(O_{2}(D)\right)=Y$ by Lemma 3.11. This is a contradiction, so (4) is proved.

Finally, since $C$ contains a $S_{2}$-subgroup of $G$, step 4 and the $Z^{*}$-theorem [6] yield $G=O(G) C$. As $2 \in \pi_{4}$ then $O(G)=1$, so $Z \triangleleft G$. But then a $S_{3}$-subgroup of $G$ normalizes a nontrivial 2-group, contradiction. Proposition 3.3 is thus proved.

Thus in order to proved Theorem 1, we are reduced to studying the case $w=2$. In this case a simple computation shows that the $S_{2}$-subgroup $T$ of $G$ has section 2-rank at most 4 , hence the theorem is a consequence of the results in Part II of [11]. However, we can avoid direct appeal to this important result by making use of prior characterizations of the groups $G_{2}(3), P S p(4,3)$ and $U_{4}(3)$ by the centralizers of their central involutions.

We retain our previous notation, so that $F=Q_{1}^{*} Q_{2}$ with $Q_{\imath} \cong Q_{8}$ and $B_{i}=C_{B}\left(Q_{i}\right), i=1,2$. We set $Z=Z(F)$ with $C=C(Z)=N(F)$. As $w=2$ then $B$ is a $S_{3}$-subgroup of $C$ and moreover $C / F \subseteq \sum_{3} \succ Z_{2}$, in particular $C=T B$ and $T / F \subseteq D_{8}$.

Now as a simple consequence of the $Z^{*}$-theorem [6] we cannot have $T=F$. Because $S C N_{3}(T) \neq \varnothing$ we also calculate that $T / F \not D_{8}$ and $T / F \not Z_{4}$. Hence $|T: F|=2^{n}$ and $T / F$ is elementary abelian, $n=1$ or 2 .

LEMma 3.12. Suppose that $A$ is an elementary abelian subgroup of $T$ with $A \triangleleft T$ and $|A|=8$. Then $A<F$.

Proof. Since $A \triangleleft T$ then $Z \leqq A_{0}=A \cap F$. Suppose that $Z=A_{0}$. Then $[A, F] \leqq Z$ so $A$ stabilizes the chain: $F \triangleright Z \triangleright 1$, so $A<F$, a contradiction. Now suppose that $\left|A_{0}\right|=4$ and choose $a \in A-A_{0}$. As a normalizes $F$ it either fixes $Q_{1}$ and $Q_{2}$ or interchanges them. If $Q_{i}^{a}=Q_{i}, i=1,2$, we get $\left[Q_{i}, a\right] \leqq Q_{i} \cap A=Z$, so a stabilizes and hence $a \in F$, contradiction. On the other hand if $Q_{1}^{a}=Q_{2}$ we get $|[F,\langle a\rangle]|=8$ against $] F,\langle a\rangle] \leqq A_{0}$. The lemma is thus proved.

Now by Lemma $2.8 G$ has a nonsolvable 2-local subgroup $N$. Lemma 3.4(b) allows us to assume that $T$ contains a $S_{2}$-subgroup of $N$. Set $D=O_{2}(N), V=\Omega_{1}(Z(D))$, and retain this notation for the 
remainder of this section. As $T$ has 2-rank at most 4 than $|V|=8$ or 16. We consider these two possibilities separately. First we have

\section{Proposition 3.4. If $|V|=8$ then $G \cong G_{2}(3)$.}

Proof. Since $C(D)=Z(D)$ we get $Z<V$, and a simple computation yields $C(V)=D$. As $N$ is not solvable we get $N / D \cong L_{3}(2)$, so $N$ has a Frobenius subgroup $K$ of order 21 transitive on $V^{*}$. Thus $K$ has a subgroup $J$ of order 3 such that $Z=C_{V}(J)$. It follows that $J<C$ and that $V=Z \times[V, J]<F$.

Now $F$ has exactly 6 elementary subgroups of order 8 , falling into two conjugacy classes of length 3 under the action of $C$. It follows that $N_{C}(V)$ contains a $S_{2}$-subgroup of $C$, hence $T<N$. Thus, $|T|=8|D|$. As $|T| \leqq 2^{7}$ then $|D| \leqq 2^{4}$ and so $D$ is abelian. Evidently $D$ cannot be nonelementary, so in fact $|D|=8, V=D$, and $|T|=2^{6}$.

Since $F<N, F \nless D$, there are involutions in $N-D$, so there is an involution $x \in N-D$ such thst $D J\langle x\rangle$ is a group with $D J\langle x\rangle / D \cong \sum_{3}$. Hence we may assume that $x$ inverts $J$, in which case $x$ centralizes $Z$. As $x \in C$ and $x \notin F$ we get $T \cong F\langle x\rangle$ and $C=F B\langle x\rangle$. Now we may assume that $J<B$. The structure of $N$ yields $J \notin \hat{B}$ (that is, $\left.C_{F}(J)=Z\right)$, so $x$ normalizes $B=O_{3}(C(J) \cap F B)$. Let $J_{1}$ be an $\langle x\rangle$ invariant complement to $J$ in $B$.

If $x$ centralizes $J_{1}$, then $x$ must interchange $Q_{1}$ and $Q_{2}$. In this case $F\langle x\rangle$ contains elementary abelian subgroups of order 16, and some such elementary subgroup of $C$ must contain $V$. But as $V=C(V)$ this is impossible. Hence, $x$ inverts $J_{1}$. Now we check that $C$ is isomorphic to the centralizer of a (central) involution of $G_{2}(3)$. By a theorem of Janko [15] we get $G \cong G_{2}(3)$ as required.

Proposition 3.5. If $|V|=16$ and $|T|=2^{6}$ then $G \cong P \operatorname{PS}(3,4)$.

Proof. As $F$ has 2-rank 3 we get $F \cap V<V$, so if $v \in V-F$ then $T=F\langle v\rangle$ and $C=F B\langle v\rangle$. Since $v$ centralizes an elementary abelian subgroup of $F$ of order 8 (namely $F \cap V$ ) then $v$ must interchange $Q_{1}$ and $Q_{2}$.

Hence $C$ is isomorphic to the centralizer of a central involution of $P S p(4,3)$. By a theorem of Janko [16] we get $G \cong P S p(4,3)$ as required.

Proposition 3.6. If $|V|=16$ and $|T|=2^{\text {T }}$ then $G \cong U_{4}(3)$.

Proof. As $V<T$ a simple computation proves that in fact $V \triangleleft T$ so that $T$ is a $S_{2}$-subgroup of $N$. Moreover $C_{T}(V)=V$, 
hence $V=C(V), V=D$, and $T / D \cong D_{8}$.

As in Proposition 3.5 we can choose $v \in V-F$. Then $v$ interchanges $Q_{1}$ and $Q_{2}$ and centralizes a subgroup $J$ of $C$ of order 3 . We may assume that $J<B$ in which case $J \notin \hat{B}$. Moreover $J$ normalizes $V \cap F=C_{F}(v)$, hence $J<N$. Let $U$ be a $S_{2}$-subgroup of $N_{C}(J)$, so that $U$ contains $\langle v, z\rangle$ as a subgroup of index 2 . We next show that $U$ is either elementary abelian or dihedral.

Since $N / D$ is nonsolvable with a dihedral $S_{2}$-subgroup of order 8 then one of the following occurs: $N / D \cong L_{2}(7), N / D \cong A_{6}$, or $N / D$ contains a subgroup isomorphic to $\sum_{5}$. Suppose to begin with that the latter case occurs. By Lemma 2.6 of [11, Part II] the extension $N / D$ splits, so $N=D H$ with $H \cong \sum_{5}$. Hence, $N \cap C=D(H \cap C)$ with $H \cap C \cong \sum_{4}$. Now $J$ is a $S_{3}$-subgroup of $N \cap C$, hence is conjugate to a $S_{3}$-subgroup of $H \cap C$, hence is inverted by a involution $x$ of $N \cap C$. We thus get that $U \cong\langle v, z, x\rangle$ has the desired isomorphism type in this case. Now suppose that $N / D$ is isomorphic to either $L_{2}(7)$ or $A_{6}$. If the $A_{6}$ case occurs then $J$ must correspond to (123) (456) since $C_{V}(J) \neq 1$ (c.f. [13, p. 157] so in either case $N_{N}(J)$ has $S_{2}$-subgroups of order 8. Moreover, since $F<N, F \nless D$ there are involutions in $N-D$, so there is an involution $x \in N-D$ which inverts $J$. Hence, $W=\langle v, z, x\rangle$ is a $S_{2}$-subgroup of $N_{N}(J)$. But $N$ contains a $S_{2}$-subgroup of $N_{C}(J)$, so $U \cong W$ and again $U$ has the required isomorphism type.

Suppose to begin with that $U$ is elementary of order 8. Then we find, since $C=F B U$, that the extension $C / F$ splits. Following a paper of Phan [22] we check that $C$ is isomorphic to the centralizer of a central involution of $L_{4}(3)$. Phan goes on to show that $G$ has a second class of involutions with nonconstrained centralizers. As $G$ is of characteristic 2-type this cannot occur, so we deduce that $U$ is not abelian.

Finally, suppose that $U \cong D_{8}$. Now we check that $C$ is isomorphic to the centralizer of an involution in $U_{4}(3)$. A second result of Phan [23] yields $G \cong U_{4}(3)$ as required. This completes the proof of Theorem 1.

4. The proof of Theorem 2. In this section we will present a proof of Theorem 2. In a sense our proof is unsatisfactory: for one thing we must assume at the outset that the relevant odd prime $p$ for which the $p$-locals are $p$-constrained is 3 . Moreover our proof utilizes several deep characterization theorems whose relevance, at least superficially, would appear to be small.

From now on, we use the following notation: $G$ is a finite simple group of characteristic 2-type and $R$ is a $S_{3}$-subgroup of $G$. We assume that all 3-local subgroups of $G$ are 3-constrained and that 
$И(R ; 2)=\{1\} . \quad B$ is an elementary subgroup of $R$ of order 9 such that $U(B ; 2) \neq\{1\}$.

Now if some 2-local subgroup of $G$ contains an element of $2(3)$ then by Theorem 1 we have $G \cong G_{2}(3), P S p(4,3)$, or $U_{4}(3)$ as required. So in trying to prove Theorem 2 we may, and shall, assume

$(*)$ No 2-local subgroup of $G$ contains an element of $\mathfrak{U}(3)$. We will eventually show that $\left(^{*}\right)$ leads to a contradiction, in which case Theorem 2 will be proved.

Our first lemma gives a number of properties of $B$ which we shall use in the sequel. First observe, since $B \notin \mathfrak{2}(3)$, that $Z(R)$ is cyclic and that $Z=\Omega_{1}(Z(R))<B$. We fix this notation for the remainder of the paper.

LEMma 4.1. The following conditions hold.

(a) If $F \in U(B ; 2)$ then $C_{F}(Z)=1$.

(b) If $F \in \boldsymbol{H}(B ; 2)$ then $F$ has class ot most 2 .

(c) $C(B)$ has odd order.

(d) $Z$ is weakly closed in $B$.

(e) If $1<B_{0}<B, B_{0} \neq Z$, then $C\left(B_{0}\right)$ is solvable.

Proof. To prove (a), let $F \in И(B ; 2)$ and suppose that $E=$ $C_{F}(Z) \neq 1$. Set $L=C_{G}(Z)>E B$. Since $Z \leqq Z(R)$ then $R<L$, hence $O_{3^{\prime}}(L)$ has odd order since $U(R ; 2)=\{1\}$. On the other hand $L$ is 3 -constrained, so there is an $E B$-invariant $S_{3}$-subgroup $P$ of $O_{3^{\prime}, 3}(L)$ on which $E$ acts faithfully. We get $\left[E, \Omega_{1}\left(C_{P}(B)\right)\right] \leqq P \cap[E, B] \leqq$ $P \cap E=1$, so $[E, P]=1$ by the generalized $P \times Q$-lemma. This is a contradiction, so (a) is proved. Part (b) follows from (a) and Lemma 2.2. As for (c), since $C(B)$ is 3-constrained and $B$ contains all elements of order 3 in $C(B)$, we find that $C(B)$ has a normal 3-complement. Now (c) follows immediately from (a).

Next choose $1 \neq F \in h(B ; 2)$. As $B$ is noncyclic there is a subgroup $1 \neq B_{0}<B$ satisfying $C_{F}\left(B_{0}\right) \neq 1$. It follows from (a) that $B_{0}$ is not conjugate to $Z$ in $G$. On the other hand, since $N_{R}(B)>C_{R}(B)$, all subgroups of order 3 in $B$ distinct from $Z$ are conjugate in $R$. Thus $Z$ is weakly closed in $B$ as required.

Finally let $1 \neq B_{0}<B$ with $B_{0} \neq Z$. Set $L=C\left(B_{0}\right)$. By (d) we can assume that $C_{R}\left(B_{0}\right)$ is a $S_{3}$-subgroup of $L$. Thus $C_{R}\left(B_{0}\right)=B_{0} \times R_{1}$ where $R_{1}$ is cyclic and $Z=\Omega_{1}\left(R_{1}\right)$. Since $L$ is 3 -constrained we get that $L$ is 3 -solvable and $L / O_{3^{\prime}}(L)$ is solvable. But by (a), $C(Z) \cap$ $O_{3^{\prime}}(L)$ has odd order. So $O_{3^{\prime}}(L)$ is solvable by Lemma 2.4 , hence $L$ is solvable, as required.

LeMma 4.2. $A S_{2}$-subgroup of $N(B)$ has order at most 2. 
Proof. Let $T$ be a $S_{2}$-subgroup of $N(B)$. We have $C_{T}(B)=1$ by Lemma 4.1(c), so $T$ acts faithfully on $B$. By Lemma 4.1(d), we have $Z \triangleleft N(B)$, so $T$ is actually both faithful and reducible on $B$. Now assume that $|T|>2$. The only possibility is $|T|=4$ and $T$ is a four-group.

Set $L=N_{G}(Z)>T B$. As usual we have $O_{3^{\prime}}(L)$ of odd order,

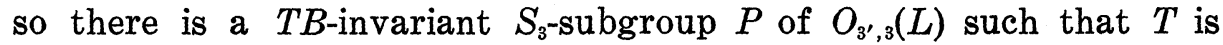
faithful on $P$. Let $K$ be a critical subgroup of $P$ of exponent 3 (that is, $K$ is a characteristic subgroup of $P$ and every $3^{\prime}$-element of $L / O_{3^{\prime}, 3}(L)$ is faithful on $K$. The existence of $K$ is proved in [9, Theorem 5.3.13]). Now as a consequence of (*) and Lemma 4.1(c) we find that if $x$ is an involution of $G$ then $C_{G}(x)$ has cyclic $S_{3}$-subgroups. Hence $|K| \leqq 27$.

Suppose first that $|K|=9$. Then $K \cong(3,3)$, so as $Z \triangleleft L$ then $L$ is solvable and $T$ covers a Hall $3^{\prime}$-subgroup of $L / O_{3^{\prime}}(L)$. Thus $L$ has a normal 2 -complement and $L$ has 3 -length 1 , so we may assume that $P=R$. Thus $K \in U(R)$, so that $K$ is contained in an element of $\operatorname{SCN}_{3}(R)$. On the other hand $K \neq B,[K, B] \neq 1$, so there is an involution of $T$ acting without fixed points on $C_{R}(K)$. This forces $C_{R}(K)$ to be abelian of rank 2 , a contradiction. So we have $|K|=27$.

Suppose $K$ is abelian. Then $K \cong(3,3,3)$, hence

$$
K=\left\langle\Omega_{1}\left(C_{K}(t)\right) \mid t \in T^{\sharp}\right\rangle>B \text {. }
$$

But then $B \in \mathfrak{A}(3)$, against (*). So $K$ must be extra-special of order 27 and exponent 3 . It follows, since Inn. $(K)=O_{3}$ (Aut. $(K)$ ), that $P=K^{*} C_{P}(K)$ and $L / O_{3^{\prime}, 3}(L) G G L(2,3)$. Next, notice that $C_{P}(K)$ is cyclic: this follows since $K \cap C_{P}(K)=Z, K=\left\langle\Omega_{1}\left(C_{K}(t)\right) \mid t \in T^{\sharp}\right\rangle$, and $C_{P}(K)$ admits $T$. Hence, we get $K=\Omega_{1}(P)$. Since $S C N_{3}(R) \neq \varnothing$, whilst $S C N_{3}(P)=\varnothing$, we get that $P<R$. As $L$ also contains a fourgroup, it follows that $L / O_{3^{\prime}, 3}(L) \cong G L(2,3)$. Now $K$ contains exactly four subgroups of order 9 , including $B$ and some $U \in U(R)$. Since $B \nsim U$ we deduce that $B$ has exactly 3 conjugates in $L$, so $N_{L}(B)$ contains a $S_{2}$-subgroup of $L$. This contradicts the first paragraph of the proof, so the lemma is proved.

Now set $R_{0}=C_{R}(B)$. As we have observed, we can assume that $R_{0}$ is a $S_{3}$-subgroup of $C_{G}(B)$. The next lemma is crucial.

Lemma 4.3. Suppose that $1 \neq F \in \mathcal{H}^{*}\left(R_{0} ; 2\right)$. Let $N=N_{G}(F)$, with $T$ a $S_{2}$-subgroup of $N$. Then the following hold:

(a) $T$ is a $S_{2}$-subgroup of $G$.

(b) $|T: F| \leqq 2$.

Proof. Let $S$ be a $S_{3}$-subgroup of $N$ which contains $R_{0}$. We may assume without loss that $S \leqq R$. Now by $\left(^{*}\right)$ we have that $S$ 
contains no element of $\mathfrak{X}(3)$, so if $U \in \mathscr{L}(R)$ we have $S \cap C_{R}(U)$ cyclic. Hence $S$ is metacyclic. Set $K=O^{3}(N)$. By a theorem of Huppert [14] $K$ has abelian $S_{3}$-subgroups. Set $S_{0}=K \cap S$.

Case 1. $S_{0}$ is non-cyclic. We get $B=\Omega_{1}\left(S_{0}\right)$, so by Lemma 4.2 it follows that $\left|N_{K}(B): C_{K}(B)\right| \leqq 2$. If $N_{K}(B)=C_{K}(B)$ then $K$ has a normal 3-complement by a transfer theorem of Burnside. If $\left|N_{K}(B): C_{K}(B)\right|=2$ then $K$ is 3 -solvable by Lemma 2.6. So in either case $K$ is 3 -solvable, hence $K=O_{3^{\prime}}(K) N_{K}(B)$. As $F$ is a $S_{2}$-subgroup of $O_{3^{\prime}}(K)$ we get $T=F N_{T}(B)$, so $|T: F| \leqq 2$ by Lemma 4.2. Finally, $T B$ is a group satisfying all the conditions of Lemma 2.3 , so $F$ is characteristic in $T$, so (a) follows and the result is proved in Case 1.

Case 2. $S_{0}$ is cyclic. If $S_{0}=1$ then $K=O_{3^{\prime}}(N)$ is the normal 3-complement of $N$, so $F=T$ and there is nothing to prove. Hence, we may assume that $S_{0} \neq 1$. Set $B_{1}=\Omega_{1}\left(S_{0}\right)<B$.

Now set $\bar{N}=N / O_{3^{\prime}}(N) . \quad C_{\bar{K}}\left(\bar{B}_{1}\right)$ has a normal 3-complement which admits $\bar{B}$, so if $C_{\bar{K}}\left(\bar{B}_{1}\right)$ has even order then there is a 2-group $\bar{U} \neq 1$ admitting $\bar{B}$. But then $B$ normalizes a $S_{2}$-subgroup of $U$ which properly contains $F$, contradiction. So $C_{\bar{K}}\left(\bar{B}_{1}\right)$ has odd order. If $\bar{K}$ has a minimal normal subgroup of order 3 then $\bar{K}$, and hence $K$, is 3 -solvable. In this case we complete the proof as in Case 1 , so we may suppose that every minimal normal subgroup of $\bar{K}$ is nonsolvable. As $\bar{K}$ has cyclic $S_{3}$-subgroup we deduce that $\bar{L}=\bar{K}^{\infty}$ is simple.

Next we have $C_{\bar{L}}\left(\bar{B}_{1}\right)$ of odd order and $\left|N_{\bar{L}}\left(\bar{B}_{1}\right): C_{\bar{L}}\left(\bar{B}_{1}\right)\right|=2$. By Lemma 2.5 we get that $\bar{L}$ has one class of involutions. Moreover, as $\bar{B}$ normalizes $N_{\bar{L}}\left(\bar{B}_{1}\right)$ there is a subgroup $B_{2}$ of order 3 in $B$ such that $\bar{B}_{2}$ centralizes an involution $\bar{x}$ of $N_{\bar{L}}\left(\bar{B}_{1}\right)$. Thus $\bar{B}_{2} \nless \bar{L}$ and $\bar{B}_{2}$ normalizes $C_{\bar{L}}(\bar{x})$. We show next that $\langle\bar{x}\rangle$ is a $S_{2}$-subgroup of $C_{\bar{L}}\left(\bar{B}_{2}\right)$. For this, it is enough to show that $C_{\bar{L}}\left(\bar{B}_{2}\right)$ is solvable: for $C_{\bar{L}}\left(\bar{B}_{2}\right)$ has a cyclic $S_{3}$-subgroup, so if $C_{\bar{L}}\left(\bar{B}_{2}\right)$ is solvable and has a $S_{2}$-subgroup of order at least 4 then $И(\bar{B} ; 2) \neq\{1\}$, a contradiction. Now if $B_{2} \neq Z$ the solvability of $C_{\bar{L}}\left(\bar{B}_{2}\right)$ follows from Lemma 4.1(e). On the other hand if $B_{2}=Z$ then $C_{G}(Z)$ has $S_{2}$-subgroups of 2-rank at most 1, so the solvability of $C_{\bar{L}}\left(\bar{B}_{2}\right)$ follows easily in this case also. So we have indeed shown that $\langle\bar{x}\rangle$ is a $S_{2}$-subgroup of $C_{\bar{L}}\left(\bar{B}_{2}\right)$.

Consider now the group $C_{\bar{L}}(\bar{x})$. It is a $3^{\prime}$-group, and moreover $C_{\bar{L}}(\bar{x}) \cap C\left(\bar{B}_{2}\right) /\langle\bar{x}\rangle$ has odd order by the last paragraph. By Lemma 2.4 we find that $C_{\bar{L}}(\bar{x}) /\langle\bar{x}\rangle$ is solvable of 2 -length 1 , so $C_{\bar{L}}(\bar{x})$ has the same property. So we have shown that $\bar{L}$ is a simple group such that every involution of $\bar{L}$ has a centralizer which is solvable of 2-length 1. Let $\bar{Y}$ be a $\bar{B}_{2}$-invariant $S_{2}$-subgroup of $\bar{L}$ with $\langle\bar{x}\rangle \leqq$ 
$Z(\bar{Y})$. We claim that $S C N_{3}(\bar{Y}) \neq \varnothing$. Otherwise, since $\langle\bar{x}\rangle=C_{\bar{Y}}\left(\bar{B}_{2}\right)$, we find easily that $\bar{Y}$ is of symplectic type, and even extra-special. But as is well-known, no simple group has such a $S_{2}$-subgroup. Thus we may now identify $\bar{L}$ using Lemma 2.9 . As $\bar{L}$ has a cyclic $S_{3}$ subgroup with an odd-order centralizer, and as $\bar{L}$ admits an automorphism (induced by $\bar{B}_{2}$ ) with fixed-point subgroup having twice odd order, we find that the only possibility is $\bar{L} \equiv L_{2}(8)$. Hence a $S_{3}$-subgroup of $N$ is isomorphic to a $S_{3}$-subgroup of Aut. $\left(L_{2}(8)\right)$, hence is metacylic of order 27 and exponent 9. But then $B=R_{0}=\Omega_{1}(S)$ char $S$ and as $\left|N_{R}(B): C_{R}(B)\right|=3$ we get $S=N_{R}(B)$. As $S C N_{3}(R) \neq \dot{\phi}$ and $B \notin \mathfrak{U}(3)$ this is impossible. So the analysis of Case 2 is completed, and the lemma is proved.

We can now prove

Proposition 4.1. $O_{3^{\prime}}\left(C\left(R_{0}\right)\right)$ is transitive on $\boldsymbol{И}^{*}\left(R_{0} ; 2\right)$.

Proof. First notice that $U\left(R_{0} ; 2\right) \neq\{1\}$. For by assumption we have $И(B ; 2) \neq\{1\}$. So if $1 \neq F \in h(B ; 2)$ and $1<B_{0}<B$ satisfies $F_{0}=C_{F}\left(B_{0}\right) \neq 1$ we get, since $F_{0} \neq Z$ by Lemma $4.1(a)$, that $C\left(B_{0}\right)$ is solvable. Thus $F_{0} \leqq\left\langle\boldsymbol{U}_{C\left(B_{0}\right)}(B ; 2)\right\rangle \leqq O_{3^{\prime}}\left(C\left(B_{0}\right)\right)$. As $R_{0}<C\left(B_{0}\right)$ then $R_{0}$ must normalize a (nontrivial) $S_{2}$-subgroup of $O_{3^{\prime}}\left(C\left(B_{0}\right)\right.$ ), as required.

Supposing the proposition false, choose elements $D_{1}, D_{2}$ in $\mathbf{U}^{*}\left(R_{0} ; 2\right)$ such that $D_{1}$ and $D_{2}$ are not conjugate in $O_{3^{\prime}}\left(C\left(R_{0}\right)\right)$ and such that $\left|D_{1} \cap D_{2}\right|$ is maximal subject to this condition. Set $D=D_{1} \cap D_{2}$. We next show that $D \neq 1$. Namely, since $D_{i} \neq 1$ then $D_{\imath}=O_{2}\left(N\left(D_{i}\right)\right)$ for $i=1,2$, and so $B$ is faithful on $D_{1}$ and $D_{2}$. Thus if $B_{i}^{*}=\{1<$ $\left.B_{0}<B \mid C_{D_{i}}\left(B_{0}\right) \neq 1\right\}$, then $\left|B_{i}^{*}\right| \geqq 2$ for $i=1,2$. As $Z \notin B_{1}, Z \notin B_{2}$ by Lemma 4.1(a) it follows that $B_{1}^{*} \cap B_{2}^{*} \neq \varnothing$. Choose $B_{0} \in B_{1}^{*} \cap B_{2}^{*}$, with $D_{i}^{*}=C_{D_{i}}\left(B_{0}\right) \neq 1, i=1,2$. As before, we get $\left\langle D_{i}^{*}, D_{2}^{*}\right\rangle \leqq O_{3^{\prime}}\left(C\left(B_{0}\right)\right)$, so there is $x \in O_{3^{\prime}}\left(C\left(R_{0}\right)\right)$ such that $\left\langle D_{1}^{*},\left(D_{2}^{*}\right)^{x}\right\rangle$ is a 2-group. It follows that $D \neq 1$.

Now set $N=N(D)$. Maximality of $|D|$ ensures that $D=O_{2}(N)$. As $N$ is 2-constrained then $C(D)=Z(D)$, in particular $Z\left(D_{i}\right) \leqq D$ for $i=1,2$. As $D_{i}$ has class at most 2 by Lemma 4.1(b), then $D \triangleleft D_{i}$, so $D_{2} \leqq N$ for $i=1,2$. Let $T_{1}$ be a $S_{2}$-subgroup of $N$ which contains $D_{1}$. By Lemma 4.3, we have $\left|T_{1}: D_{1}\right| \leqq 2$. Moreover $D_{1} / D$ is abelian, so $T_{1} / D$ has an abelian subgroup of index at most 2 .

As in Lemma 4.3, we may argue that $N$ has metacyclic $S_{3}$-subgroup. Suppose that $N$ is 3 -solvable. Then we get

$$
\left\langle N_{D_{1}}(D), N_{D_{2}}(D)\right\rangle \leqq\left\langle\boldsymbol{И}_{N^{\prime}}\left(R_{0} ; 2\right)\right\rangle \leqq O_{3^{\prime}}(N),
$$

so $\left\langle N_{D_{1}}(D), N_{D_{2}}(D)^{x}\right\rangle$ is a 2-group for some $x \in O_{3},\left(C\left(R_{0}\right)\right)$, and the maximality of $|D|$ is contradicted. Hence, $N$ is not 3 -solvable. The 
argument of Case 1 of the previous lemma now yields that $K=O^{3}(N)$ has a nontrivial cyclic $S_{3}$-subgroup, and moreover if $\bar{N}=N / O_{3^{\prime}}(N)$ then $\bar{L}=\bar{N}^{\infty}$ is simple. Now as $\bar{N}$ has $S_{2}$-subgroups which have abelian subgroups of index at most $2, \bar{L}$ has the same property, so we may identify $\bar{L}$ using Lemma 2.10. In particular, if $T=T_{1} \cap L$, so that $\bar{T}$ is a $S_{2}$-subgroup of $\bar{L}$, then $\bar{T}$ is either elementary abelian, or isomorphic to $D_{2} n, S D_{2} n$ or $Z_{2} n<Z_{2}$. Now $B$ normalizes $D_{1} \cap L$ and $\left|T: D_{1} \cap L\right| \leqq 2$. As $B \cap L$ is cyclic it follows that there is a subgroup $B_{0}$ of $B$ of order 3 such that $B_{0} \nless L$ and $B_{0}$ normalizes a $S_{2}$-subgroup of $L$. Hence we can assume that $B_{0}$ normalizes $T$.

Now if $T$ is non-abelian then $\left[T, B_{0}\right]=1$. Since $C_{L}(B)$ has odd order by Lemma $4.1(\mathrm{c})$ it follows in this case that $C_{\bar{L}}\left(\bar{B}_{1}\right)$ has odd order, where $B_{1}=\Omega_{1}(B \cap L)$. It follows that $\bar{L} \not L_{3}(q)$ or $U_{3}(q)(q$ odd), $A_{7}$ or $M_{11}$, for these groups have nonabelian $S_{2}$-subgroups and elements of order 6 .

Suppose we have $\bar{L} \cong L_{2}(q)$ for some $q$. We may choose $B_{2}<B$, $\left|B_{2}\right|=3, B_{2} \neq Z, B_{2} \nless L$. Then $B_{2}$ induces a field automorphism of $\bar{L}$ of order 3 . Since $C\left(B_{2}\right)$ is solvable by Lemma $4.1(\mathrm{e})$, the only possibilities are $q=8$ or $q=27$. In the latter case $\bar{L}$ has a noncyclic $S_{3}$-subgroup, a contradiction. We may eliminate the former possibility as in Lemma 4.3 , and so $\bar{L} \not L_{2}(q)$ for any $q$.

As $J_{1}$ has no outer automorphisms of order 3 [17], and as groups of Ree-type have non-cyclic $S_{3}$ subgroups [18], $\bar{L}$ can be isomorphic to none of these groups. Having exhausted the possibilities given by Lemma 2.10 , we deduce that $\bar{L}$ does not exist, so the proof of Proposition 4.1 is completed.

The Proof of Theorem 2. We retain the notation of the previous lemma, so that $B \leqq R_{0}=C_{R}(B)$. As $S C N_{3}(R) \neq \varnothing$ then there is $U \in \mathscr{U}(R)$ such that $R_{0} U=N_{R}(B)$. So $\left|R_{0} U: R_{0}\right|=3$, so $R_{0} \triangleleft R_{0} U$. Hence, $U$ must permute the elements of $U^{*}\left(R_{0} ; 2\right)$ among themselves. By Proposition $4.1 \boldsymbol{U}^{*}\left(R_{0} ; 2\right)$ contains a $3^{\prime}$ number of elements, so $U$ must fix some element $F \in \mathcal{U}^{*}\left(R_{0} ; 2\right)$. As $F \neq 1$ then $И(U ; 2) \neq\{1\}$. However, $U \in \mathfrak{N}(3)$. This contradicts our basic assumption $\left(^{*}\right)$, so Theorem 2 is proved.

\section{REFERENCES}

1. J. L. Alperin and R. Lyons, On conjugacy classes of p-elements, J. Algebra, 19 (1971), 536-537.

2. H. Bender, Über den grössten $p^{\prime}$-Normalteiler in p-auflöbaren Gruppen, Arch. Math., 18 (1967), 15-16.

3. R. Brauer, Some applications of the theory of blocks of characters of finite groups III, J. Algebra, 3 (1966), 225-255.

4. W. Feit, and J. G. Thompson, Solvability of groups of odd order, Pacific J. Math., 13 (1963), 775-1029. 
5. R. Gilman and D. Gorenstein, Finite groups with Sylow 2-subgroups of class two I, II (to appear).

6. G. Glaubermann, Central elements in core-free groups, J. Algebra, 4 (1966), 403420 .

7. D. Gorenstein, On finite groups of characteristic 2-type, Inst. Hautes Etudes Sci. Publ. Math., 36 (1969), 5-13.

8. Centralizers of involutions in finite simple groups (in) Finite Simple Groups, Proceedings of London Math. Soc. Conf., London: Academic Press, 1971.

9. Finite Groups, Harper and Row, New York, 1968.

10. - A program for classifying simple groups, Lecture notes, University of Chicago (unpublished).

11. D. Gorenstein and K. Harada, Finite groups whose 2-subgroups are generated by at most 4 elements, (to appear).

12. G. Higman, Odd characterizations of finite simple groups, Lecture notes, University of Michigan, 1968.

13. B. Huppert, Endliche Gruppen I, Springer-Verlag, Berlin, 1967.

14. - Gruppen mit modularer Sylow-Gruppe, Math. Z., 75 (1961), 140-153.

15. Z. Janko, A characterization of the finite simple group $G_{2}(3)$, J. Algebra, 12 (1969), 360-371.

16. - A Characterization of the finite simple group PSp(4,3), Canada. J. Math., 19 (1967), 872-894.

17. - A new finite simple group with abelian $S_{2}$-subgroups and its characterization, J. Algebra, 4 (1966), 147-186.

18. and J. G. Thompson, On a class of finite simple groups of Ree, J. Algebra, 4 (1966), 274-292.

19. K. Klinger and G. Mason, Centralizers of p-groups in groups of characteristic 2, p-type, (to appear).

20. R. J. Lundgren, On finite simple groups all of whose 2-local subgroups are solvable, J. Algebra, 27 (1973), 491-515.

21. G. Mason, The characterization of finite groups whose Sylow 2-subgroup has a cyclic commutator subgroup, (to appear).

22. K.-W. Phan, A characterization of the finite simple group $L_{4}(3)$, J. Austr. Math. Soc., X (1969), 51-76.

23. - A characterization of the finite simple group $U_{4}(3)$, J. Austr. Math. Soc., X (1969), 77-95.

24. S. D. Smith and A. D. Tyrer, On finite groups with a certain Sylow normalizer II, J. Algebra, 26 (1973), 366-367.

25. M. Suzuki, On a class of doubly transitive groups, Ann. Math., 75 (1962), 105-146. 26. J. G. Thompson, Non-solvable finite groups all of whose local subgroups are solvable, I.: Bull. Amer. Math. Soc. 74 (1968), 383-437; II: Pacific J. Math., 33 (1970), 451-537; III: Pacific J. Math., 39 (1971), 483-534; IV: Pacific J. Math., 48 (1973), 511-592; balance to appear.

27. J. H. Walter, The characterization of finite groups with abelian Sylow 2-subgroups, Ann. Math., 89 (1969), 405-514.

Received September 13, 1974. Partially supported by NSF GP-42875.

University of California, Santa Cruz 



\title{
PACIFIC JOURNAL OF MATHEMATICS
}

\author{
EDITORS
}

RICHARD ARENS (Managing Editor)

University of California

Los Angeles, California 90024

R. A. Beaumont

University of Washington

Seattle, Washington 98105
J. DugundJI

Department of Mathematics University of Southern California Los Angeles, California 90007

D. Gilbarg and J. Milgram

Stanford University

Stanford, California 94305

\section{ASSOCIATE EDITORS}

E. F. BECKENBACH

B. H. NeUManN

F. WOLF

K. YosHIDA

\section{SUPPORTING INSTITUTIONS}

UNIVERSITY OF BRITISH COLUMBIA

GALIFORNIA INSTITUTE OF TECHNOLOGY

UNIVERSITY OF CALIFORNIA

MONTANA STATE UNIVERSITY

UNIVERSITY OF NEVADA

NEW MEXICO STATE UNIVERSITY

OREGON STATE UNIVERSITY

UNIVERSITY OF OREGON

OSAKA UNIVERSITY
UNIVERSITY OF SOUTHERN CALIFORNIA

STANFORD UNIVERSITY

UNIVERSITY OF TOKYO

UNIVERSITY OF UTAH

WASHINGTON STATE UNIVERSITY

UNIVERSITY OF WASHINGTON

AMERICAN MATHEMATICAL SOCIETY NAVAL WEAPONS CENTER 


\section{Pacific Journal of Mathematics}

\section{Vol. 57, No. $1 \quad$ January, 1975}

Keith Roy Allen, Dendritic compactification ......................... 1

Daniel D. Anderson, The Krull intersection theorem ..................... 11

George Phillip Barker and David Hilding Carlson, Cones of diagonally dominant

matrices .............................................

David Wilmot Barnette, Generalized combinatorial cells and facet splitting ........

Stefan Bergman, Bounds for distortion in pseudoconformal mappings ............

Nguyên Phuong Các, On bounded solutions of a strongly nonlinear elliptic

equation ...............................................

Philip Throop Church and James Timourian, Maps with 0-dimensional critical

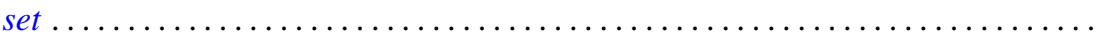

G. Coquet and J. C. Dupin, Sur les convexes ubiquitaires ................. 67

Kandiah Dayanithy, On perturbation of differential operators............... 85

Thomas P. Dence, A Lebesgue decomposition for vector valued additive set

functions .............................................. 91

John Riley Durbin, On locally compact wreath products.................. 99

Allan L. Edelson, The converse to a theorem of Conner and Floyd ............. 109

William Alan Feldman and James Franklin Porter, Compact convergence and the

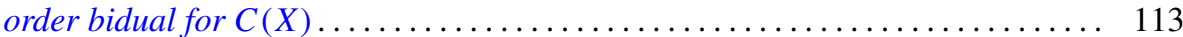

Ralph S. Freese, Ideal lattices of lattices......................... 125

R. Gow, Groups whose irreducible character degrees are ordered by divisibility ... 135

David G. Green, The lattice of congruences on an inverse semigroup ............ 141

John William Green, Completion and semicompletion of Moore spaces .......... 153

David James Hallenbeck, Convex hulls and extreme points of families of starlike and close-to-convex mappings .................................. 167

Israel (Yitzchak) Nathan Herstein, On a theorem of Brauer-Cartan-Hua type ...... 177

Virgil Dwight House, Jr., Countable products of generalized countably compact spaces ............................................ 183

John Sollion Hsia, Spinor norms of local integral rotations. I . . . . . . ......... 199

Hugo Junghenn, Almost periodic compactifications of transformation

semigroups....................................

Shin'ichi Kinoshita, On elementary ideals of projective planes in the 4-sphere and oriented $\Theta$-curves in the 3 -sphere ............................ 217

Ronald Fred Levy, Showering spaces............................ 223

Geoffrey Mason, Two theorems on groups of characteristic 2-type............. 233

Cyril Nasim, An inversion formula for Hankel transform ................... 255

W. P. Novinger, Real parts of uniform algebras on the circle ................ 259

T. Parthasarathy and T. E. S. Raghavan, Equilibria of continuous two-person

games............................................... 265

John Pfaltzgraff and Ted Joe Suffridge, Close-to-starlike holomorphic functions of several variables ....................................... 271

Esther Portnoy, Developable surfaces in hyperbolic space .................. 281

Maxwell Alexander Rosenlicht, Differential extension fields of exponential type . . . 289

Keith William Schrader and James Lewis Thornburg, Sufficient conditions for the

existence of convergent subsequences ........................... 301

Joseph M. Weinstein, Reconstructing colored graphs .................. 307 